This item was submitted to Loughborough's Research Repository by the author.

Items in Figshare are protected by copyright, with all rights reserved, unless otherwise indicated.

\title{
The biomechanics of twisting somersaults. Part III: aerial twist
}

PLEASE CITE THE PUBLISHED VERSION

PUBLISHER

(C) Taylor \& Francis

VERSION

AM (Accepted Manuscript)

LICENCE

CC BY-NC-ND 4.0

REPOSITORY RECORD

Yeadon, Maurice R.. 2019. "The Biomechanics of Twisting Somersaults. Part III: Aerial Twist". figshare. https://hdl.handle.net/2134/5321. 
This item was submitted to Loughborough's Institutional Repository (https://dspace.lboro.ac.uk/) by the author and is made available under the following Creative Commons Licence conditions.

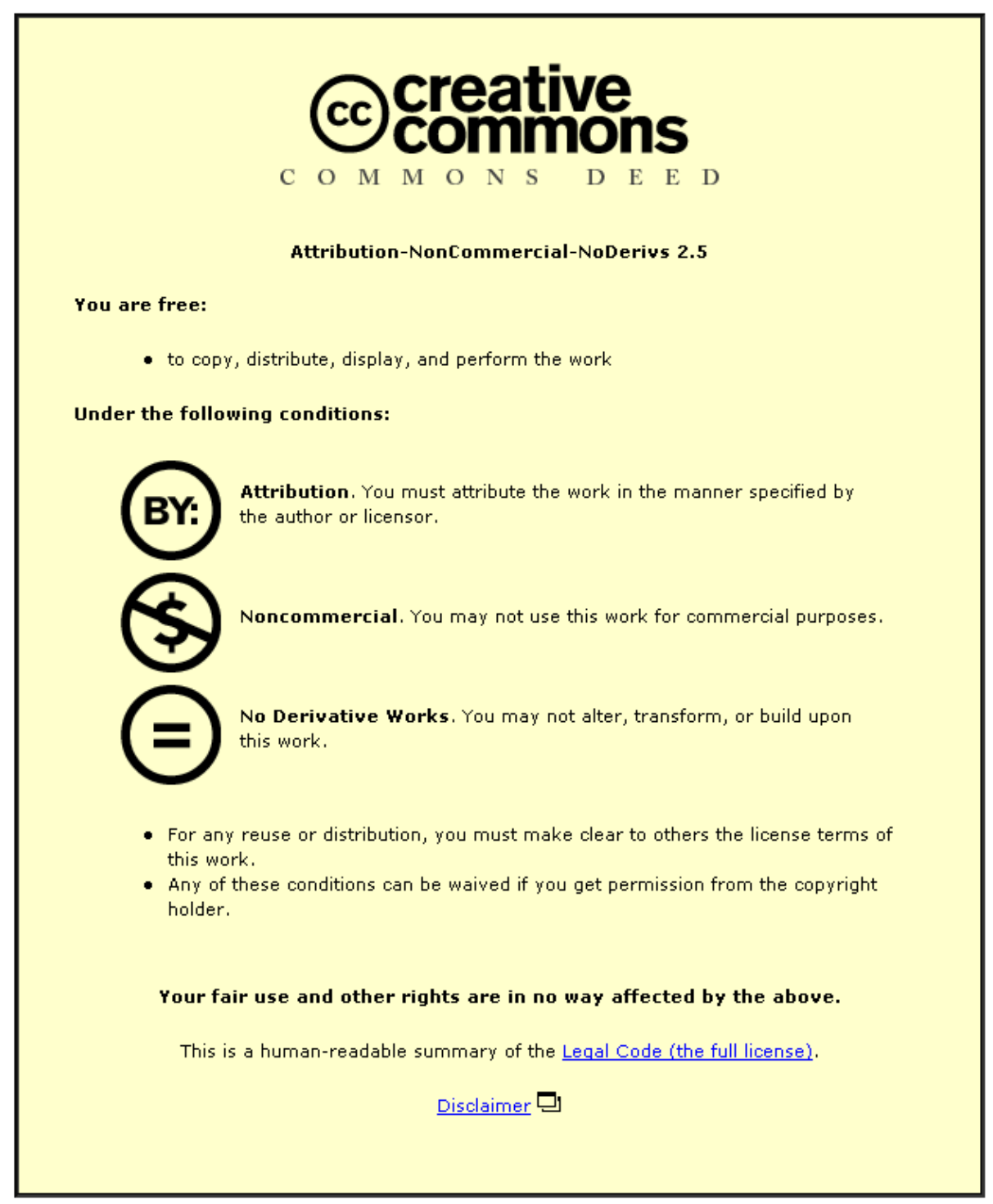

For the full text of this licence, please go to: http://creativecommons.org/licenses/by-nc-nd/2.5/ 


\title{
The biomechanics of twisting somersaults. Part III: Aerial twist
}

\author{
M.R. YEADON* \\ Sports Biomechanics Laboratory, Department of Physical Education and Sports Science, \\ Loughborough University, Ashby Road, Loughborough LE11 3TU, UK \\ and Human Performance Laboratory, University of Calgary, Canada
}

\begin{abstract}
A simulation model and a rigid body model are used to evaluate aerial twisting techniques. It is found that when somersault is not present, a number of cycles of segment counter-rotation are required to produce one twist. When somersault is present, twist may be introduced by producing tilt using asymmetrical movements of the arms, chest or hips about the sagittal plane. The same asymmetrical movements may be used to remove tilt, although the effectiveness of these techniques is dependent upon body configuration and the direction of somersault.
\end{abstract}

Keywords: Twist, somersault, model, simulation, biomechanics.

\section{Introduction}

In Part II of this series of papers, techniques which initiated twist during the contact phase were evaluated. In Part III, techniques for producing twist after takeoff will be considered.

The fact that a cat can twist over when dropped in an inverted position demonstrates that it is possible for an animal to produce twist while airborne (McDonald, 1960). Theoretical investigations into astronaut reorientation under conditions of zero angular momentum have indicated that humans can produce whole body rotations using counter-rotations of the arms or legs. The amounts of twist that can be produced by such segmental movements are small, however. It has been found that one cycle of arm movement can produce $33^{\circ}$ of twist (Kane and Scher, 1970), while one cycle of arm and leg movement can produce $54^{\circ}$ of twist. Counter-rotation of the hips will result in twist, since the angular momentum associated with the hip movement must be balanced by a rotation of the whole body in the opposite direction (Van Gheluwe, 1981). There is experimental evidence that it is possible to produce up to $1 \frac{1}{4}$ twists using this method (McDonald, 1961). Whether it is possible to produce multiple twists using this method is open to question.

In this paper, twist produced under conditions of zero angular momentum will be referred to as counter-rotation twist. Other terms for counter-rotation twist include 'action-reaction twist' (LaDue and Norman, 1967), 'contrary-motion twist' (Eaves, 1969), 'non-inertial twist' (Kosa and Kamimura, 1972) and 'torque-free twist with zero angular momentum' (Frohlich, 1979).

When somersault is present so that the angular momentum is not zero, there is the possibility of using this angular momentum to produce twist. By raising one arm sideways and lowering the other, the body of a diver will become tilted out of the somersault plane so that there will be a component of angular momentum along the twist axis (Batterman, 1974). Scher and Kane (1969) used a two-segment model to show that it is theoretically possible to convert a pure twist into a pure somersault by repeatedly abducting and adducting one arm. Pike (1980) showed that a full twist may be introduced into a plain dive by means of asymmetrical arm movements.

In addition to the use of asymmetrical arm movements, it has been proposed that twisting the shoulders when the body is piked will also produce tilt (Rackham, 1970; Batterman, 1974). It is of interest to enquire how effective such techniques are for the production of tilt and how much twist will result. Since the angular momentum is used for twist when the body is tilted, Barrow (1959) held

\footnotetext{
*Address all correspondence to M. R. Yeadon, Department of Physical Education and Sports Science, Loughborough University, Ashby Road, Loughborough LE11 3TU, UK.
} 
the view that the introduction of twist will slow the somersault, whereas Bunn (1972) stated that the somersault rate is increased whenever twist is present. Rackham (1970) reasoned that the moment of inertia about the somersault axis is decreased when there is tilt so that the somersault rate will increase. Thus it is not clear what effect the introduction of twist does have on the somersault rate.

Twist produced during a somersault will be referred to as tilt twist. Other terms for tilt twist include 'twist by somersault transfer' (Rackham, 1970) and 'twist by trading momentum' (Hay, 1985). In this paper, the ability of counter-rotation and tilt techniques to produce twist will be investigated using the 11-segment simulation model of Yeadon et al. (1990) and the rigid body model developed in Part I.

\section{Counter-rotation twist}

When a counter-rotation technique is employed, the body twists only so long as the segments are being counter-rotated. Thus it is important to establish how much twist can result from one cycle of counterrotation, since the method is limited by the number of cycles that can be performed in the time available. The angular momentum $\mathbf{h}$ of a system of linked rigid segments may be expressed in the form:

$$
\mathbf{h}=\mathbf{h}_{f}+\mathbf{h}_{\mathrm{rel}}
$$

where $\mathbf{h}_{f}$ is the angular momentum due to the motion of the system frame $f$ and $\mathbf{h}_{\text {rel }}$ is the angular momentum due to movements of the segments relative to frame $f$ (Yeadon, 1990).

If the whole body twists through an angle $\psi$ as a result of the counter-rotation of body segments through an angle $-\psi_{s}$, then:

$$
h_{f}=I_{f} \dot{\psi} \quad \text { and } \quad h_{\mathrm{rel}}=I_{s} \dot{\psi}_{s}
$$

where $I_{f}$ is the moment of inertia of the whole body about the twist axis and $I_{s}$ is an inertia term associated with the internal movement. If the total angular momentum is zero, the equation of motion takes the form:

$$
I_{f} \dot{\psi}-I_{s} \dot{\psi}_{s}=0
$$

As an example, consider equation (6) which is developed later for counter-rotation of the hips:

$$
\text { (6) : } \quad C \psi-\left[C-\left(C_{1} \cos \alpha_{1}+C_{2} \cos \alpha_{2}\right) \sec \delta\right] \psi_{p}=0
$$

Clearly, $I_{f}=C$ is greater than $I_{s}=C-\left(C_{1} \cos \alpha_{1}+C_{2} \cos \alpha_{2}\right) \sec \delta$.

In general, the inertia terms $I_{f}$ and $I_{s}$ will vary as the configuration changes but the whole body inertia $I_{f}$ will always be greater than the segmental term $I_{s}$ and so the twist angle $\psi$ will be less than the counter-rotation angle $\psi_{s}$. This result places a restriction on all counter-rotation techniques and shows that in order to produce multiple twists it is necessary to use a number of cycles of counter-rotation.

An example is given by the simulation CR1 shown in Fig. 1, where a half twist is produced by one cycle of hip rotation when there is an angle of $130^{\circ}$ between trunk and legs.

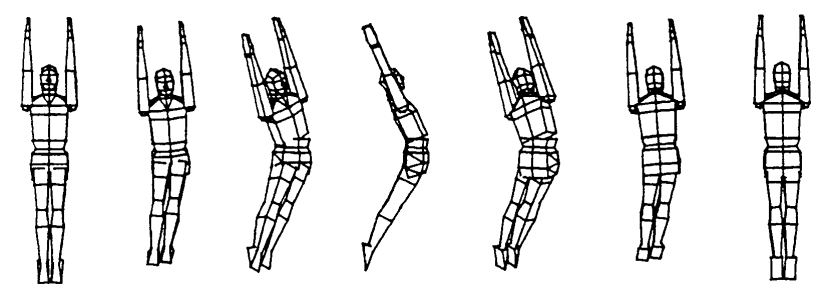

Figure 1: Twist produced by counter-rotation of the hips (CR1).

\section{Tilt twist}

Suppose that a gymnast is performing a plain somersault with the lateral axis $\mathbf{f}_{1}$ parallel to the horizontal angular momentum vector $\mathbf{h}$. If tilt is introduced so that the motion is transformed into a somersault with a sustained twist, the twist rate $\psi$ will be given by equation (4) in Part I as: 


$$
\dot{\psi}=(h / C-\dot{\phi}) \sin \theta
$$

where $\theta$ is the angle between the twist axis and the vertical plane normal to $\mathbf{h}, \dot{\phi}$ is the somersault rate and $C$ is the principal moment of inertia about the twist axis.

For a sustained twist, the motion must be in the twisting mode and the tilt angle $\theta$ will vary between the values $\alpha$ and $\beta$ (see Part I). The motion will change from a plain somersault to a somersault with sustained twist only when the tilt angle is changed from zero and so the term tilt twist is appropriate.

Since the angular momentum vector is horizontal, the apparent angle of tilt between the twist axis and the vertical somersault plane is identical to the tilt angle between the twist axis and the plane normal to the angular momentum vector, whereas for contact twist a distinction has to be made between the two angles.

Any configurational change in which symmetry about a sagittal plane is not maintained will produce tilt. The ability of arm, chest and hip movements to produce tilt will be evaluated.

\section{Rotations of the arms}

If the total angular momentum is zero, then abduction of the left arm will produce a tilting of the whole body in the opposite direction as shown in simulation TL1 (Fig. 2a). Since the tilt is produced by counter-rotation of the arm, the equation of motion will have the same form as equation (1):

$$
I_{f} \dot{\theta}-I_{a} \dot{\epsilon}_{a}=0
$$

where $\theta$ is the angle of tilt of the mid-line $\mathbf{f}_{3}$ of the body, $\epsilon_{a}$ is the abduction angle of the left arm, $I_{f}$ is the whole body moment of inertia about the frontal axis $\mathbf{f}_{2}$, and $I_{a}$ is the inertia term associated with the arm movement.
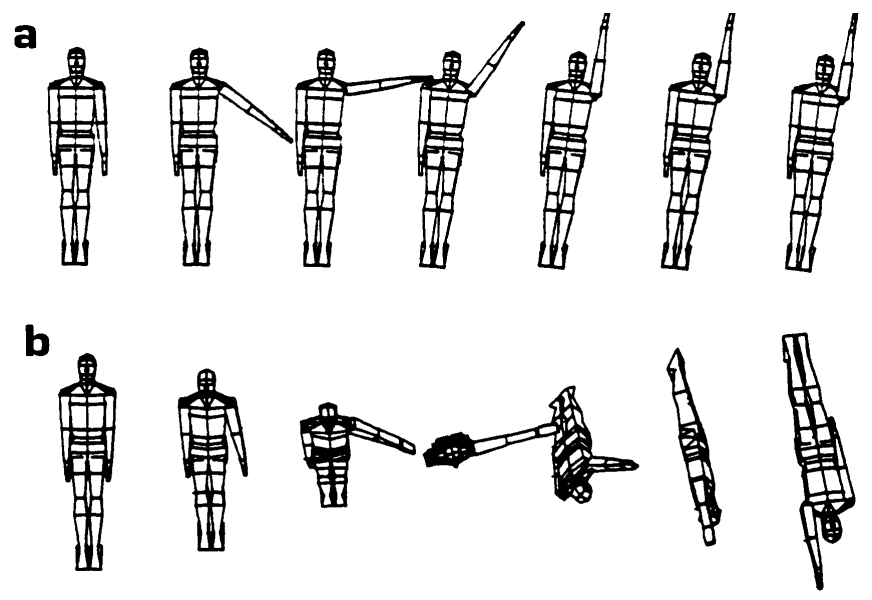

Figure 2: Production of tilt using asymmetrical arm movement in (a) a straight jump (TL1) and (b) a straight forward somersault (TL2).

Table 1 shows how the tilt angle $\theta$ increases with the arm abduction angle $\epsilon_{a}$. As a result of the arm asymmetry, the principal axis corresponding to minimum moment of inertia makes an angle $\theta_{p}$ with axis $\mathbf{f}_{3}$. Thus the tilt of the principal axis is given by $\beta=\theta+\theta_{p}=8.6^{\circ}$. Using the inertia parameters of two other subjects gives values of $8.5^{\circ}$ and $7.1^{\circ}$ for angle $\beta$.

If the same arm movement is made instantaneously during a somersault, then the same angle of tilt will result. For a slower arm movement, the nutation effect will occur and this will increase the tilt angle during the first quarter twist. However, as the twist angle increases, the plane of arm movement moves away from the vertical somersault plane and less tilt is produced. In the following example, maximum use is made of the nutation effect but the second half of the arm movement is ineffective.

Suppose that during a somersault the left arm is rapidly abducted through $90^{\circ}$ so that the total tilt is $\beta=5.1^{\circ}$ (Table 1). The nutation effect will increase the tilt from $\beta$ to $\alpha$, which are related by equation 
Table 1: Tilt produced by asymmetrical arm movement, TL1

\begin{tabular}{cccccc}
\hline$\epsilon_{a}$ & $\theta$ & $\theta_{p}$ & $I_{1}$ & $I_{f}$ & $I_{3}$ \\
\hline $0^{\circ}$ & $0.0^{\circ}$ & $0.0^{\circ}$ & 10.56 & 11.03 & 0.72 \\
$30^{\circ}$ & $0.0^{\circ}$ & $0.5^{\circ}$ & 10.58 & 11.32 & 0.99 \\
$60^{\circ}$ & $0.5^{\circ}$ & $1.8^{\circ}$ & 10.72 & 11.77 & 1.29 \\
$90^{\circ}$ & $1.5^{\circ}$ & $3.6^{\circ}$ & 11.09 & 12.24 & 1.40 \\
$120^{\circ}$ & $3.1^{\circ}$ & $4.4^{\circ}$ & 11.63 & 12.62 & 1.23 \\
$150^{\circ}$ & $4.9^{\circ}$ & $3.6^{\circ}$ & 12.11 & 12.81 & 0.94 \\
$180^{\circ}$ & $6.9^{\circ}$ & $1.7^{\circ}$ & 12.28 & 12.75 & 0.71 \\
\hline
\end{tabular}

$\epsilon_{a}$, abduction angle of left arm; $\theta$, angle of tilt of mid-line of body; $\theta_{p}$, additional tilt due to arm asymmetry; $I_{f}$, principal moment of inertia about axis $\mathbf{f}_{2} ; I_{3}$, minimum principal moment of inertia.

(16) of Part 1 :

$$
(1 / C-1 / A) \cos ^{2} \alpha=(1 / C-1 / B) \cos ^{2} \beta
$$

Using the values for the principal moments $A, B$ and $C$ given in Table 1 results in $\alpha=8.4^{\circ}$ at the quarter twist position. If the left arm is abducted rapidly through a further $90^{\circ}$ at the quarter twist position, the tilt angle will not change but will fall to $\beta_{1}=7.9^{\circ}$ at the half twist position as a negative nutation effect occurs. Although the nutation effect has increased the tilt from $5.1^{\circ}$ to $7.9^{\circ}$, the final tilt angle is less than the value $\beta=8.6^{\circ}$ produced by a rapid $180^{\circ}$ arm abduction. However, the fact that the gain in tilt from the nutation effect is offset by the decreased effectiveness of the arm movement means that the tilt angle is not particularly sensitive to the duration of the arm movement.

In simulation TL2, the arm movement takes place throughout the first half somersault (Fig. 2b) and results in a tilt angle of $\beta=9.3^{\circ}$, which is greater than the $8.6^{\circ}$ produced by a rapid arm movement and the $7.9^{\circ}$ produced by a delayed arm movement. This indicates that maximum tilt will be produced by an arm movement that is of intermediate duration rather than by a rapid or slow arm movement. The maximum possible tilt will lie between $\beta=9.3^{\circ}$ and $\beta=8.6^{\circ}+2.8^{\circ}=11.4^{\circ}$, where the last figure has been obtained by adding the gain in tilt during the delayed movement to the tilt produced by a rapid arm movement.

Since the response of tilt to the duration of arm movement is relatively flat, the particular arm movement which produces maximum tilt is of minor interest, whereas it is of importance that an arm movement of almost any duration will produce about $9^{\circ}$ of tilt. This lack of sensitivity will tend to make the technique easy to learn and execute.

Since different tilt techniques will be compared using the values of the tilt angle $\beta$ for an elite trampolinist, it will be helpful to have a corresponding measure of the twist rate. The number of twists per somersault may be calculated approximately using equation (25) developed in Part I for a rod:

$$
p / \Omega=(I / C-1) \sin \theta
$$

where $p / \Omega$ is the number of twists per somersault, $I$ is the average of the principal inertias $A$ and $B, C$ is the principal moment of inertia about the twist axis and $\theta$ is the average angle of tilt.

The average tilt angle $\theta$ may be taken as the average of the extreme values $\alpha$ and $\beta$. If the body is straight, either with both arms by the sides or with one arm overhead, $\theta$ may be approximated as $\theta=\beta+0.3^{\circ}$ with less than $1 \%$ error in the range $7^{\circ}<\beta<20^{\circ}$. For the asymmetrical arm position used in simulations TL1 and TL2, the ratio $I / C=17.6$, whereas for the position with both arms adducted, $I / C=15.0$. Using these values in equation (3) gives Table 2.

The oscillation effect of the wobbling mode may be used to increase the tilt angle as discussed for contact twist (see Part II). This technique is used in simulation TL3 in which the arm moves in the plane of the trunk (Fig. 3). When the body is piked and the right arm is abducted in this plane, not only is tilt produced but there is also a small counter-rotation twist to the right. Since in a backward somersault the tilt twist produced by this arm action is to the left, it is clear that the tilt angle will rise 
Table 2: Twist rates for different tilt angles

\begin{tabular}{ccc}
\hline Tilt angle & \multicolumn{2}{c}{ No. of twists per somersault } \\
\cline { 2 - 3 }$(\beta)$ & $\begin{array}{c}\text { Asymmetrical } \\
\text { arms }\end{array}$ & $\begin{array}{c}\text { Symmetrical } \\
\text { arms }\end{array}$ \\
\hline $8^{\circ}$ & 2.4 & 2.0 \\
$12^{\circ}$ & 3.5 & 3.0 \\
$16^{\circ}$ & 4.7 & 3.9 \\
$20^{\circ}$ & 5.8 & 4.9 \\
\hline
\end{tabular}

Notes: For the asymmetrical arm position, the abduction angles of the arms are $0^{\circ}$ and $180^{\circ}$. For the symmetrical arm position, the abduction angles of the arms are $0^{\circ}$.

until the twist angle reaches the zero position again. The extension is made from the pike while the tilt angle is near its maximum and a value of $\beta=14.0^{\circ}$ is obtained.

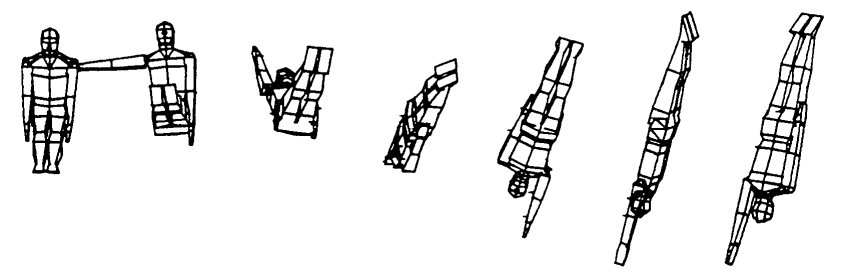

Figure 3: Production of tilt using asymmetrical arm movement in a piked backward somersault (TL3).

As the body extends from the pike, the motion changes from the wobbling mode to the twisting mode. Until the zero twist position is reached, the tilt angle will continue to rise due to the oscillation effect in the wobbling mode and the nutation effect in the twisting mode. These effects are essentially the same in the neighbourhood of $\psi=0$ and are governed by equation (15) of Part I which may be written as:

$$
\cos ^{2} \alpha=\cos ^{2} \theta\left(1-\sin ^{2} \psi \sin ^{2} \alpha_{0}\right)
$$

where $\alpha$ is the final angle of tilt at $\psi=0, \theta$ is the tilt produced by the arm movement, $\psi$ is the counterrotation twist produced by the arm movement and $\alpha_{0}$ is the critical $\alpha$-value and is a function of the pike position.

In order for the increase in tilt to be large, it is necessary to have a large angle $\psi$ of counter-rotation twist. The arm movement of simulation TL3 produces a counter-rotation twist of only $15^{\circ}$ and, although this is increased since the asymmetric arm position reorients the directions of the principal axes, the gain in tilt is only about $2^{\circ}$. If the time taken for the arm movement and extension from the pike is increased from that of simulation TL7, the resulting tilt remains close to $\beta=14.0^{\circ}$ even when the duration is increased by a factor of 3 .

If the same arm movement is used in a forward somersault, the direction of twist produced by counterrotation of the arm is the same as the direction of twist resulting from the tilt. In this situation, the oscillation of the tilt angle is past the peak and has started to fall so that time spent in the piked position has a detrimental effect on the tilt angle. If the timing of the internal movement is identical to that of simulation TL3, the resulting tilt is $\beta=8.7^{\circ}$ compared with $14.0^{\circ}$ in a backward somersault. If the duration of internal movement is increased by a factor of 3 , the tilt angle produced in a forward somersault falls to zero.

In order to exploit the nutation and oscillation effects in a piked forward somersault, it will be necessary to use an arm movement which produces a counter-rotation twist in the direction opposite to the tilt twist. Thus the optimum plane of arm movement is dependent upon the direction of the somersault. 


\section{Rotation of the chest}

In order to obtain maximum tilt using the wobbling mode oscillation effect, the counter-rotation twist angle $\psi$ should approach $90^{\circ}$. If the chest is twisted through $60^{\circ}$ relative to the thorax with arms abducted at $90^{\circ}$, the counter-rotation twist is about $30^{\circ}$, but since the asymmetrical position of the chest and arms causes a reorientation of the principal axes, the effective counter-rotation twist is more than $60^{\circ}$. The tilt angle produced for the elite trampolinist by such a movement is $5.5^{\circ}$, while values of $5.0^{\circ}$ and $5.4^{\circ}$ are obtained using the segmental inertia parameters of two other subjects.

If the same movement is made during a forward somersault, the counter-rotation twist will be in the opposite direction to the twist resulting from the tilt so that the oscillation effect will increase the tilt angle. This is shown in simulation TL4, where the tilt angle has been increased from $5.5^{\circ}$ to $\beta=14.0^{\circ}$ (Fig. 4a). If the duration of the internal movement is increased by a factor of 2 , the resulting tilt angle is $20^{\circ}$. The maximum possible tilt angle $\alpha$ may be calculated from equation (4):

$$
\cos ^{2} \alpha=\cos ^{2} \theta\left(1-\sin ^{2} \psi \sin ^{2} \alpha_{0}\right)
$$

The maximum value of $\alpha_{0}$ during the movement is $21^{\circ}$ and the maximum possible value of $\sin \psi$ is 1 . Taking $\theta=5.5^{\circ}$ produces $\alpha=21.7^{\circ}$.

a

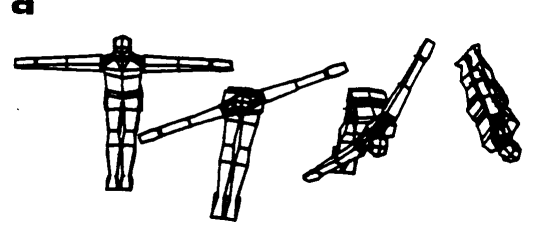

b

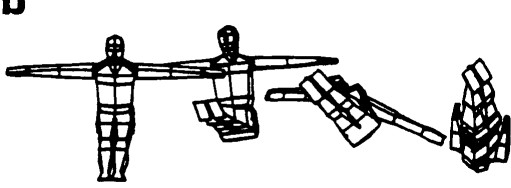

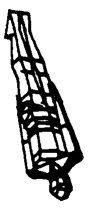
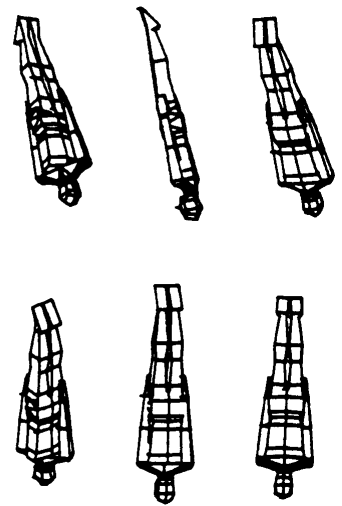

Figure 4: Production of tilt using torsion of the chest in (a) a piked forward somersault (TL4) and (b) a piked backward somersault (TL5).

In simulation TL5, the internal movement of simulation TL4 is used in a backward somersault. Since the direction of counter-rotation twist is the same as the direction of tilt twist, the tilt angle has passed its peak value in the oscillation cycle and is falling rapidly. As a consequence, the tilt produced is near zero and very little twist occurs (Fig. 4b). In order to use this technique effectively in a backward somersault, it is necessary to start from an arched rather than a piked position.

\section{Rotation of the hips}

As shown by Kane and Scher (1969), the hula movement of the hips may be modelled using two equal cylinders. While such a model is adequate for the description of counter-rotation twist, the assumed symmetry of the model implies that no tilt is produced during the movement. To investigate this, a model comprising two unequal cylinders will be used. If tilt can be produced, then another aerial twisting technique will have been identified.

Let $\mathbf{u}_{3}$ and $\mathbf{1}_{3}$ be the axes of symmetry of the upper cylinder $U$ and lower cylinder $L$ and let $\mathbf{e}_{3}$ be the whole body principal axis corresponding to minimum moment of inertia (Fig. 5). Let $\mathbf{e}_{2}$ be the whole body principal axis lying in the pike plane $\mathbf{u}_{3} \mathbf{1}_{3}$ so that the reference frame $e$ moves with the pike plane.

If the cylinders $U$ and $L$ have angular velocities $\mathbf{n}_{u}$ and $\mathbf{n}_{l}$ about their own axes relative to frame $e$ and the magnitudes of $\mathbf{n}_{u}$ and $\mathbf{n}_{l}$ are each $n=\psi_{p}$, the angular momentum associated with this movement 


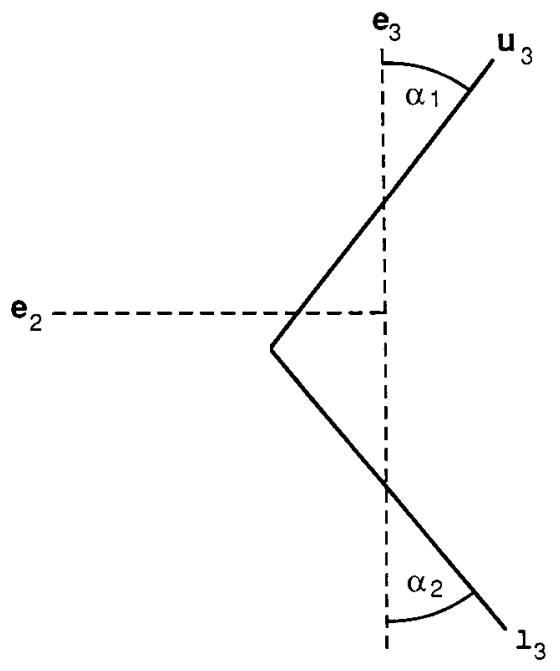

Figure 5: The geometry of the axes $\mathbf{u}_{3}, \mathbf{1}_{3}$, and $\mathbf{c}_{3}$, of a hula movement.

will be (in frame $e$ ):

$$
\mathbf{h}_{\mathrm{rel}}=\left[\begin{array}{ccc} 
& 0 & \\
-C_{1} n \sin \alpha_{1} & + & C_{2} n \sin \alpha_{2} \\
C_{1} n \cos \alpha_{1} & + & C_{2} n \cos \alpha_{2}
\end{array}\right]
$$

where $C_{1}$ and $C_{2}$ are the principal inertias of $U$ and $L$ about their axes of symmetry and $\alpha_{1}$ and $\alpha_{2}$ are the angles $\mathbf{u}_{3}$ and $\mathbf{l}_{3}$ make with $\mathbf{e}_{3}$.

If the system counter-rotates at a rate of $\omega$ about the inertial axis $\mathbf{i}_{3}$, which lies in the pike plane (Fig. 6), the associated angular momentum in frame $\mathbf{e}$ will be:

$$
\mathbf{h}_{e}=\left[\begin{array}{ccc}
A & 0 & 0 \\
0 & B & 0 \\
0 & 0 & C
\end{array}\right] \cdot\left[\begin{array}{c}
0 \\
\omega \sin \delta \\
-\omega \cos \delta
\end{array}\right]
$$

where $A, B$ and $C$ are the whole body moments of inertia about $\mathbf{e}_{1}, \mathbf{e}_{2}$ and $\mathbf{e}_{3}$, and $\delta$ is the angle between $\mathbf{i}_{3}$ and $\mathbf{e}_{3}$.

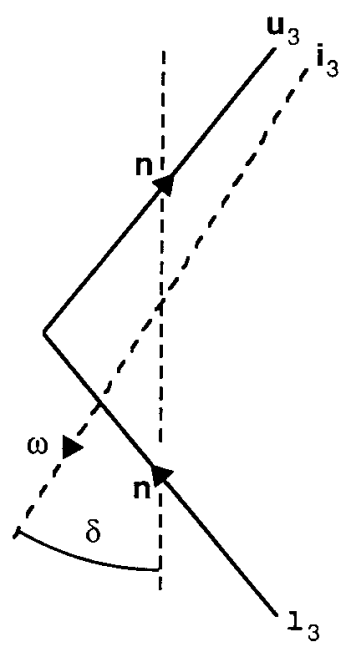

Figure 6: Angular velocities $\mathbf{n}_{u}, \mathbf{n}_{l}$, and $\omega$ during a hula movement.

If the total momentum $\mathbf{h}_{e}+\mathbf{h}_{\mathrm{rel}}$ is zero, then:

$$
\begin{aligned}
& B \omega \sin \delta=\left(C_{1} \sin \alpha_{1}-C_{2} \sin \alpha_{2}\right) n \\
& C \omega \cos \delta=\left(C_{1} \cos \alpha_{1}+C_{2} \cos \alpha_{2}\right) n
\end{aligned}
$$


so that:

$$
\begin{aligned}
& \tan \delta=C\left(C_{1} \sin \alpha_{1}-C_{2} \sin \alpha_{2}\right) / \\
& \quad B\left(C_{1} \cos \alpha_{1}+C_{2} \cos \alpha_{2}\right)
\end{aligned}
$$

As a result of the internal movement, the system rotates about $\mathbf{i}_{3}$ at rate $\omega=\dot{\psi}_{e}$ and the angle of tilt between axis $\mathbf{e}_{3}$ and the initial orientation of the pike plane will reach its maximum value of $\delta$ when $\psi_{e}=90^{\circ}$. The amount of twist of the body frame $f$ may then be obtained as:

$$
\psi=\psi_{p}-\psi_{e}=\psi_{p}-90^{\circ}
$$

where $\dot{\psi}_{p}=n$ and $\dot{\psi}_{e}=\omega$ so that:

$$
C \psi-\left[C-\left(C_{1} \cos \alpha_{1}+C_{2} \cos \alpha_{2}\right) \sec \delta\right] \phi_{p}=0
$$

The tilt angle $\delta$ will be large when the pike is deep so that $C / B$ and $\alpha_{1}$ are large and when the arms are abducted so that $C_{1}$ is large.

In simulation TL6, a quarter hula movement is made with an arm abduction angle of $60^{\circ}$ and a pike angle of $130^{\circ}$ (Fig. 7a-d). In the second phase of the movement, the arms are adducted and the body is extended from the piked position (Fig. $7 \mathrm{~d}-\mathrm{g}$ ). This extension from the pike reduces the tilt angle by $7.8^{\circ}$ to $2.0^{\circ}$.

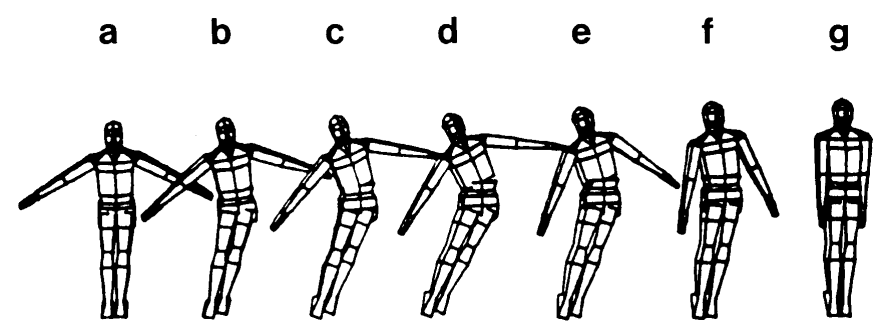

Figure 7: A partial hula movement with extension (TL6).

If the hula movement is made during a forward somersault, the direction of twist due to the tilt will be the same as the direction of the counter-rotation twist. However, when the body is in the side pike position (Fig. 7d), the lateral body axis $\mathbf{f}_{1}$ is aligned with the principal axis corresponding to the intermediate principal moment of inertia. Thus the tilt angle has yet to reach its maximum value due to the nutation effect and the twist angle of the principal axis reference frame is effectively $\psi_{1}=\psi-90^{\circ}-68^{\circ}$. Using equation (4):

$$
\cos ^{2} \alpha=\cos ^{2} \theta\left(1-\sin ^{2} \psi_{1} \sin ^{2} \alpha_{0}\right)
$$

with $\theta=9.8^{\circ}, \psi_{1}=-68^{\circ}$ and $\alpha_{0}=10.7^{\circ}$ gives a maximum tilt angle of $\alpha=14.9^{\circ}$ at the quarter twist position when $\psi=90^{\circ}$. If extension from the pike is made at this time, the tilt angle will not change and in the subsequent motion in the twisting mode the tilt angle will vary between $\alpha=14.9^{\circ}$ and $\beta=14.5^{\circ}$.

If the extension from the pike is made between the quarter and half twist positions, the tilt angle will have fallen due to the nutation effect, but this will be offset by an increase in the tilt angle due to the extension from the pike. If extension is made when $\psi=180^{\circ}-22^{\circ}=158^{\circ}$, the tilt angle will have fallen again to $9.8^{\circ}$ due to the nutation effect and will increase by $7.8^{\circ}$ upon extension to $\theta=17.6^{\circ}$. The cancelling of the two effects means that the timing of the extension is not critical and so long as extension is made between $\frac{1}{4}$ and $\frac{3}{4}$ twists, the resulting tilt angle will be around $\beta=16^{\circ}$.

In simulation TL7, the hula movement is made while the body is extending from a $90^{\circ}$ pike during a forward somersault so that, after a quarter of a hula cycle, the body is in a side pike position with pike angle $\gamma=130^{\circ}$. The extension from the side pike is made between the twist values 0.21 and $0.69 \mathrm{rev}$ and the resulting tilt angle is $\beta=17.5^{\circ}$ (Fig. 8a).

If the hula movement is made during a backward somersault, the twist due to the tilt will be in the opposite direction and so the tilt angle will be falling rather than rising. In simulation TL8, the internal movements of TL7 are made during a backward somersault. During the hula movement the 

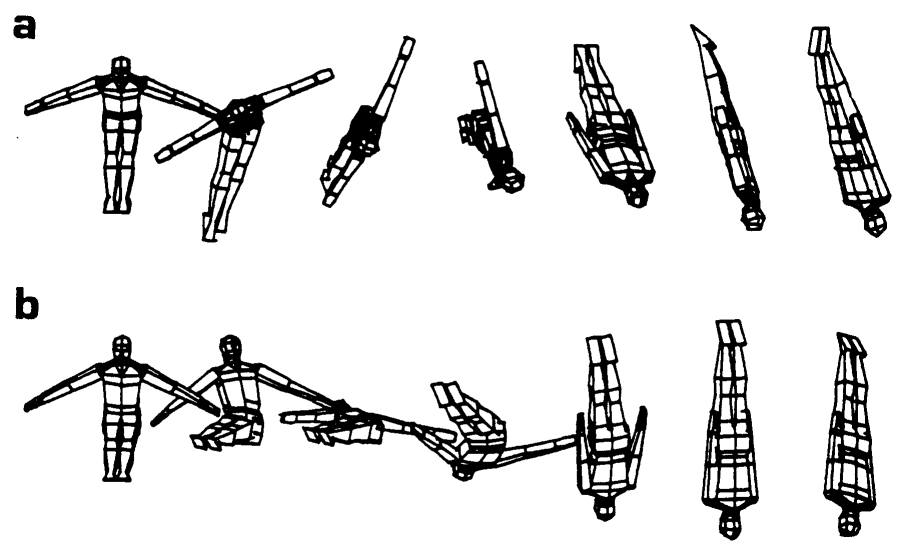

Figure 8: Production of tilt using a quarter hula cycle in (a) a piked forward somersault (TL7) and (b) a piked backward somersault (TL8).

counter-rotation twist is to the right while the twist arising from the tilt is to the left so that, when the side pike position is reached, the twist angle is close to zero (Fig. 8b). Although the tilt angle is about $8^{\circ}$ at this time, the subsequent extension reduces the angle of tilt to near zero since the twist angle is near zero. As a consequence, less than half a twist is produced during the somersault.

If the side pike position were to be held until the quarter twist position is reached, the subsequent extension would result in a tilt angle of about $16^{\circ}$. In a backward somersault such a delay becomes excessive, since the body will have rotated through a complete somersault by the time the extension has been made. Thus the hula movement is much more effective in producing tilt during a piked forward somersault than during a piked backward somersault.

If the body is somersaulting backwards in a back arched position, then a partial hula movement to a side pike position will produce tilt twist in the same direction as the twist resulting from the counterrotation. As a consequence, the quarter twist position is reached without undue delay and effective use can be made of the nutation effect.

\section{Stopping the twist}

It has been shown that a plain somersault may be transformed into a sustained twisting somersault by producing tilt using rotations of the arms or chest or using a hula movement of the hips. Each of these techniques is also capable of removing the tilt and examples may be obtained by considering simulations which produce tilt to be run backwards in time. Since the total angular momentum remains constant, the reversed movements are as valid as the original simulations and show that a twisting somersault may be transformed into a plain somersault by using any of the tilt-producing techniques to remove the tilt.

If asymmetrical arm movements are used to produce and remove tilt, then for an even number of half twists the final arm movement will be the reverse of the original arm movement. Thus in forward somersault dives with $1,2,3$ or 4 twists, it is advantageous to leave one arm above the head during the twist, in preparation for the final arm movement. In the case of an odd number of half twists, the arm action required to remove the tilt is the same as that required to produce the tilt and so it is a disadvantage to maintain the original asymmetrical arm configuration.

Since torsion of the chest, while extending from a pike, is effective in producing tilt only in a forward somersault, the reverse movement will be effective in the removal of tilt only when the somersault rotation is backwards prior to landing, since the body will be moving from a straight to a piked position. In the same way, the hula movement is effective in producing tilt from a pike when the initial somersault direction is forwards and is effective in removing tilt when the final somersault direction is backwards. It should be noted that during a twist in the straight position, the value of the hula angle is notional and the configuration is symmetrical. If the hula movement is used to produce and remove tilt in a movement, which initially somersaults forwards and comprises an odd number of half twists, the direction of hula movement will be the same at the beginning and end of the movement. In each case, the counter-rotation 
twist produced by the hula movement will be in the same direction as the body is twisting and so the direction of hula movement will be the natural one.

Tilt may also be removed by piking sufficiently so as to make use of the oscillation effect in the wobbling mode. As the tilt angle approaches zero, extension may be made from the pike so that the twisting mode of motion applies and the tilt angle will remain small.

\section{The somersault rate}

The effect on the somersault rate when a tilt twist is introduced into a somersault will now be determined. Equation (2) from Part I gives the somersault rate as:

$$
\dot{\phi}=h\left(\cos ^{2} \psi / I_{1}+\sin ^{2} \psi / I_{2}\right)
$$

where $\psi$ is the angle of twist, $h$ is the angular momentum and $I_{1}$ and $I_{2}$ are the moments of inertia about the principal axes $\mathbf{f}_{1}$ and $\mathbf{f}_{2}$.

Prior to the production of tilt the somersault rate will be $h / I_{1}$ since the twist angle will be zero. After the production of tilt the somersault rate will vary between $h / I_{1}$ and $h / I_{2}$ with an average value of approximately $h / I$, where $I$ is the average of $I_{1}$ and $I_{2}$.

If the body is in the straight position with arms adducted, $I_{2}$ will be greater than $I_{1}$ so that the somersault rate will be smaller during the twist. For the trampolinist, $I_{1}=10.56 \mathrm{~kg} \cdot \mathrm{m}^{2}$ and $I_{2}=$ $11.01 \mathrm{~kg} \cdot \mathrm{m}^{2}$, so that the somersault rate will decrease by about $2 \%$. It should be noted that this decrease is practically independent of the angle of tilt. Thus while additional twist in a somersault implies a greater tilt angle, the somersault rate will not be affected.

\section{Discussion}

When somersault is not present, twist may be produced using counter-rotation of the arms or hips. The amount of twist that can be produced by such techniques is limited, however, since it requires a number of cycles of counter-rotation to produce a full twist. A single cycle of hip movement is capable of producing a half twist providing there is sufficient flexion between the trunk and legs. It may be expected that this type of aerial twist will be used in movements such as the half-out piked on trampoline, in which a half twist is produced in the second somersault. On the other hand, counter-rotation twist is unlikely to make a large contribution to somersaults with multiple twists.

When somersault is present, twist may be introduced by producing a tilt of the body away from the vertical somersault plane. Movements of the arms, chest or hips which are asymmetrical about the sagittal plane will result in such tilt. All three methods are capable of producing twist rates in excess of four twists per somersault. Asymmetrical arm movement is effective in both straight and piked backward and forward somersaults, whereas chest and hip movements are more effective from a pike in forward somersaults and from an arch in backward somersaults. In order that advantage may be taken of the nutation effect, the tilt-producing technique should also result in a counter-rotation twist so that the tilt angle will rise. This means that the arm movement in a piked backward somersault should lie close to the plane of the trunk, whereas in a piked forward somersault it should be in front of the chest. It is to be expected that these techniques will be used in movements such as the full-out or rudi-out, in which the twist occurs within the second somersault. Since all three methods are capable of producing rapid multiple twists, the particular method used may vary from individual to individual.

Twist may be completely stopped by reducing the tilt angle to zero. This may be done using the appropriate asymmetrical movement of the arms, chest or hips. As in the production of tilt, asymmetrical arm movement may be used to remove tilt in both forward and backward somersaults, whereas chest and hip movements are only effective when the somersault direction is backwards prior to landing. Thus in trampoline and tumbling skills, it may be expected that some use will be made of chest and hip movement in the removal of tilt, whereas in twisting dives only asymmetrical arm movement is likely to be used, since the direction of somersault prior to entry is forwards. The removal of tilt is an important aspect of performance in trampolining, since not only must the twist be controlled prior to landing but the sagittal plane should be close to vertical upon landing. If this is not so, the trampolinist will have 
to make additional adjustments during the contact phase and may have difficulty with the subsequent skill.

It has been shown that the introduction of tilt twist into a straight somersault decreases the somersault rate by only $2 \%$. In Part II, it was shown that the introduction of contact twist into a straight somersault changed the somersault rate by a similar small amount. Thus the controversy on how the somersault rate is affected by the introduction of twist may be resolved by saying that the effect is small. The changes in body configuration associated with the change from a nontwisting somersault to a twisting somersault may have more bearing on the situation. In a single backward somersault, the arms are likely to be held lower in the twisting somersault, which will rotate further until an adjustment is made to the takeoff. When a piked double somersault is modified by introducing twist into the second somersault, the extension from the pike is likely to occur earlier compared with the non-twisting movement. As a consequence, the twisting double will require additional somersault momentum to be produced during takeoff. The implication of these considerations is that appropriate lead-up movements are those which exhibit similar configurational changes to the desired twisting movement.

While the speculation upon which techniques are likely to be used is based upon a sound theoretical foundation, the matter can only be resolved by analysing actual performances of twisting somersaults. In Part IV, a method is presented for partitioning twisting performances into contributions from contact and aerial techniques.

\section{References}

Barrow, C. H. (1959). Diving coaching. Swimming Times, 36(6):184-186.

Batterman, C. (1974). The Techniques of Springboard Diving. MIT Press, Cambridge, Mass.

Bunn, J. W. (1972). Scientific Principles of Coaching. Prentice-Hall, Englewood Cliffs, N.J.

Eaves, G. (1969). Diving: The Mechanics of Springboard and Firmboard Techniques. Kaye and Ward, London.

Frohlich, C. (1979). Do springboard divers violate angular momentum conservation? American Journal of Physics, 47:583-592.

Hay, J. G. (1985). The Biomechanics of Sports Techniques. Prentice-Hall, Englewood Cliffs, N.J.

Kane, T. R. and Scher, M. P. (1969). A dynamical explanation of the falling cat phenomenon. International Journal of Solids and Structures, 5:663-670.

Kane, T. R. and Scher, M. P. (1970). Human self-rotation by means of limb movement. Journal of Biomechanics, 3:39-49.

Kosa, F. and Kamimura, M. (1972). A study of mechanism of non-inertial twist. Bulletin of Institute of Sport Science, 10:69-81.

LaDue, F. and Norman, J. (1967). Two Seconds of Freedom. Nissen Trampoline Company, Brentwood.

McDonald, D. (1960). How does a cat fall on its feet? New Scientist, 7:1647-1649.

McDonald, D. (1961). How does a man twist in the air? New Scientist, 10:501-503.

Pike, N. L. (1980). Computer simulation of a forward, full twisting dive in a layout position. PhD thesis, Pennsylvania State University.

Rackham, G. W. (1970). The fascinating world of twist: Twist by somersault transfer. Swimming Times, 47:267-263.

Scher, M. P. and Kane, T. R. (1969). Alteration of the state of motion of a human being in free fall. Technical report, Division of Applied Mechanics, Stanford University, Stanford, Calif. Technical Report No. 198, NASA-CR 108938. 
Van Gheluwe, B. (1981). A biomechanical simulation model for airborne twist in backward somersaults. Journal of Human Movement Studies, 7:1-22.

Yeadon, M. R. (1990). The simulation of aerial movement - III. The determination of the angular momentum of the human body. Journal of Biomechanics, 23:75-83.

Yeadon, M. R., Atha, J., and Hales, F. D. (1990). The simulation of aerial movement - IV. A computer simulation model. Journal of Biomechanics, 23:85-89. 